\section{Migration transendothéliale au niveau de l'unité neurovasculaire}

\author{
«Une coupe pour traverser la barrière »
}

Sylvie Cazaubon, Nicolas Weiss, Pierre-Olivier Couraud
Institut Cochin, Inserm U1016, CNRS UMR8104, département de génétique et développement, 24, rue du faubourg Saint-Jacques, 75014 Paris, France; université Paris Descartes, Sorbonne Paris Cité Paris, 75014 France.

sylvie.cazaubon@inserm.fr
La barrière hémato-encéphalique ou unité neurovasculaire

La barrière hémato-encéphalique (BHE), située anatomiquement à l'interface entre le sang et le parenchyme cérébral, contrôle les échanges entre ces deux compartiments et participe au maintien de l'homéostasie cérébrale. Historiquement, l'existence d'une barrière entre le sang et le cerveau a été suggérée par les travaux d'Ehrlich en 1885, puis démontrée par plusieurs études dont celles de Lewandowski (1900) qui auraient proposé le terme de «blut-hirn-schranke», barrière sang-cerveau, et de Goldmann (1913). Considérée initialement comme une barrière statique, celle-ci va pro- gressivement apparaître comme une barrière dynamique, et c'est en 1967 que Reese et Karnovsky localisèrent la fonction de barrière au niveau des cellules endothéliales $(C E)$ cérébrales qui, par la présence de jonctions intercellulaires serrées, forment une ceinture continue ou zonula occludens (Figure IA). Les jonctions serrées permettent un accolement ou kiss point, visible en microscopie électronique (forte densité aux électrons), des membranes plasmiques des deux cellules endothéliales adjacentes et délimitent le pôle apical ou luminal (lumière du vaisseau) et le pôle basal ou abluminal (face au parenchyme cérébral) (Figure 1C).
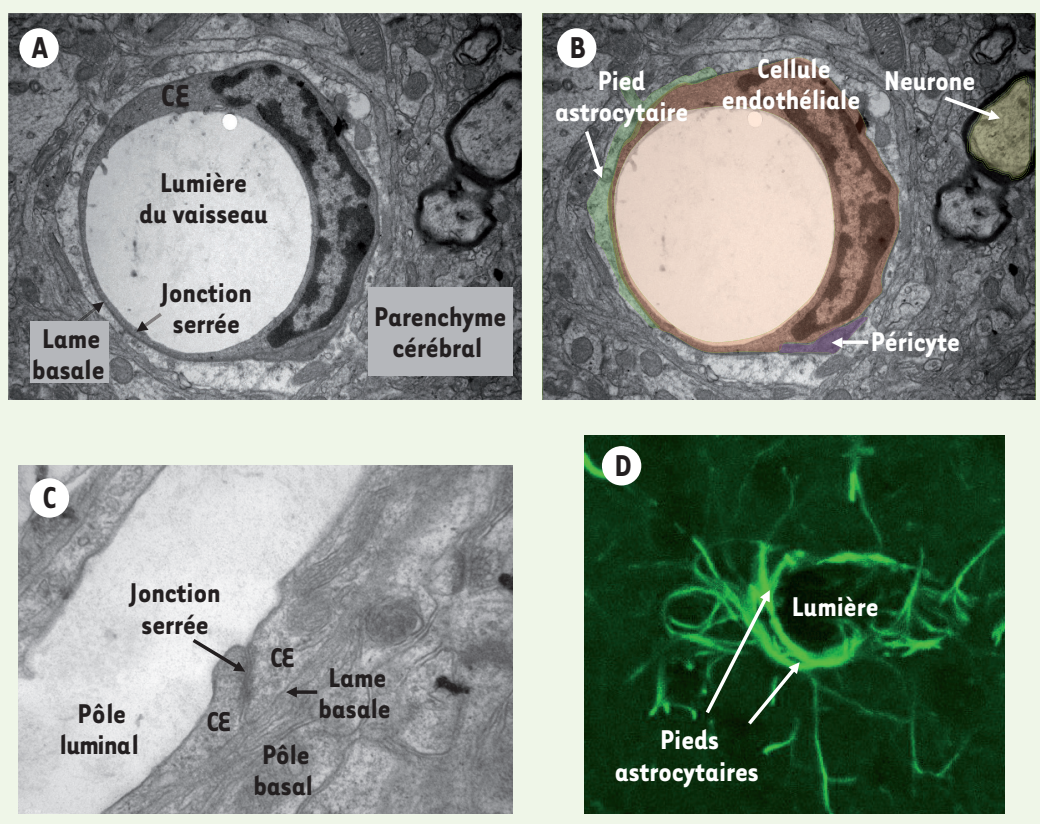

Depuis, de nombreuses études ont démontré que l'acquisition et le maintien de l'intégrité structurale et fonctionnelle de la BHE sont sous le contrôle des cellules périvasculaires. À leur pôle basal, les cellules endothéliales cérébrales sont en contact avec la lame basale constituée de deux feuillets, l'un produit par les cellules endothéliales, l'autre par les astrocytes périvasculaires qui entourent les péricytes (Figure IB). Le rôle des péricytes dans la régulation du débit sanguin et le maintien d'une faible perméabilité de la BHE est connu depuis plusieurs années. Plus récemment, des travaux ont révélé que les péricytes participent aussi à la mise en place des jonctions serrées au cours du développement, et donc à la maturation de la BHE [1]. Cette dernière fonction a longtemps été attribuée essentiellement

Figure 1. L'unité neurovasculaire. $A, B$. Vue en microscopie électronique d'un microvaisseau de cerveau de rat en coupe coronale illustrant les différents types cellulaires constituant l'unité neurovasculaire : cellule endothéliale cérébrale (brun), péricyte (violet), astrocyte (vert) et neurone (jaune). C. Vue en microscopie électronique d'une jonction serrée entre deux cellules endothéliales (CE) adjacentes, visualisée grâce à sa forte densité aux électrons. D. Reconstruction en 3D d'un microvaisseau de cerveau de rat entouré de pieds astrocytaires. L'immunomarquage de la GFAP (glial fibrillary acidic protein, marqueur des astrocytes, en vert) est analysé en microscopie confocale. 

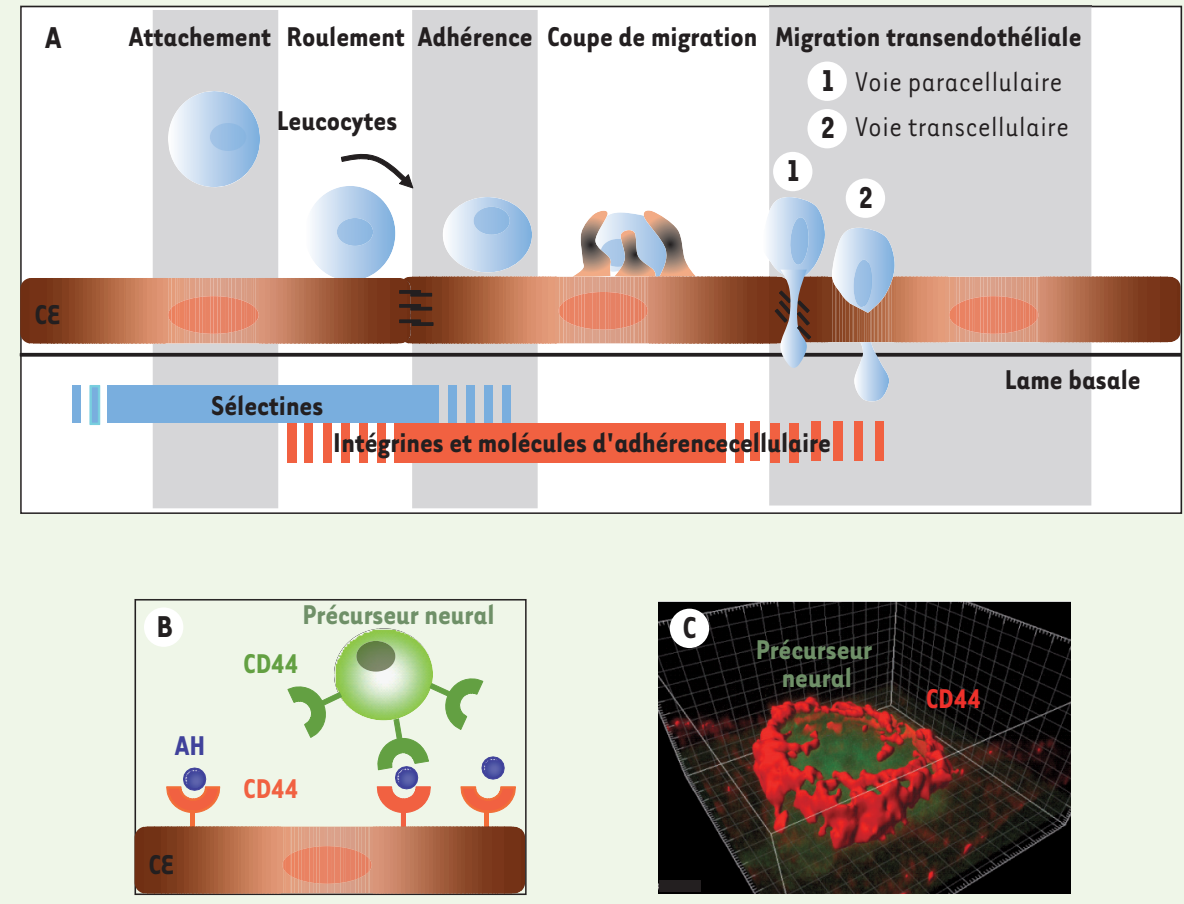

Figure 2. La migration transendothéliale. A. Représentation schématique des différentes étapes de la migration transendothéliale et des molécules impliquées. B. Représentation schématique de l'implication de CD44 via l'acide hyaluronique $(\mathrm{AH})$ dans l'interaction entre la cellule circulante (leucocyte, cellule cancéreuse métastatique, ou précurseur neural) et la cellule endothéliale (CE). $C$. Reconstitution en 3D d'une coupe de migration induite par un précurseur neural (en vert) à la surface d'une monocouche de cellules endothéliales cérébrales, immunofluorescence de CD44 (en rouge) acquise en microscopie confocale.

aux astrocytes, cellules étoilées avec de longs prolongements cellulaires (ou pieds astrocytaires) entourant les microvaisseaux cérébraux sur l'ensemble de leur surface (Figure 1D). Les astrocytes périvasculaires et les péricytes, voire les cellules endothéliales cérébrales elles-mêmes, sont également en contact avec des projections neuronales (Figure IB), ce qui permet aux neuromédiateurs de réguler le débit sanguin régional et probablement aussi de moduler les échanges sang-cerveau [12]. Ce couplage neurovasculaire met en jeu de nombreux mécanismes qui ont fait l'objet d'études récentes et révèlent le rôle prédominant des astrocytes [2]. L'ensemble de ces observations a conduit à revoir la notion de BHE et le terme d'unité neurovasculaire ou «neurovascular unit » est désormais utilisé pour mieux rendre compte du rôle des différents acteurs intervenant dans cette interface [3, 4].

\section{La migration transendothéliale}

L'entrée dans le cerveau de cellules présentes dans la circulation sanguine est très finement régulée au niveau de l'unité neurovasculaire. En situation physiologique, la surveillance immunitaire du cerveau est assurée par l'infiltration, à travers l'endothélium cérébral, d'un nombre limité de leucocytes. Ce n'est qu'en situation inflammatoire qu'une entrée importante de leucocytes est observée, situation qui permet aussi la migration transendothéliale de cellules non leucocytaires dont la présence dans la circulation est anormale. Tel est le cas de certaines cellules tumorales métastatiques provenant de tumeurs primaires périphériques (cancer du sein, cancer du poumon à petites cellules) [5].
En outre, la migration de cellules souches (cellules souches hématopoïétiques, précurseurs neuraux), administrées dans la circulation sanguine lors de stratégies de thérapie cellulaire, a été documentée $[6,7]$.

Le processus de migration transendothéliale des leucocytes, qui a été bien étudié au niveau des organes périphériques, l'est encore de façon incomplète au niveau de l'unité neurovasculaire. En périphérie, il se déroule en plusieurs étapes successives (I'attachement, le roulement, l'adhérence et la migration) qui font intervenir des molécules d'adhérence leucocytaires et leurs contre-récepteurs endothéliaux (sélectines, intégrines et molécules d'adhérence cellulaire) (Figure 2A) [3, 13]. En situation inflammatoire, l'adhérence et la migration leucocytaires sont stimulées par une augmentation de l'expression et/ou de l'activité des molécules d'adhérence cellulaire sur les deux types cellulaires, leucocytes et cellules endothéliales, favorisant ainsi la migration des leucocytes activés. D'autres cellules présentes dans la circulation et qui traversent aussi l'endothélium cérébral (cellules tumorales métastatiques, précurseurs neuraux), bien qu'avec moins d'efficacité que les leucocytes, ont en commun d'exprimer la molécule d'adhérence leucocytaire CD44 (S. Cazaubon, observations personnelles et [8]). L'acide hyaluronique (AH) présent au niveau de l'arborisation glycosylée du pôle luminal des cellules endothéliales est un ligand de CD44 et permet le pontage entre deux molécules CD44, l'une présente à la membrane des cellules endothéliales, l'autre 
exprimée par les cellules tumorales métastatiques ou les précurseurs neuraux (Figure 2B).

\section{Formation d'une coupe de migration} L'expression des molécules d'adhérence cellulaire à la surface de la cellule circulante est capable d'induire, au cours de l'étape d'adhérence, la formation d'une « coupe de migration » via la réorganisation de la membrane luminale de la cellule endothéliale. Cette coupe est formée par de longs prolongements membranaires émis par les cellules endothéliales, qui vont entourer la cellule adhérente (Figure 2C). Ces prolongements endothéliaux sont très riches en molécules d'adhérence cellulaire (ICAM-1 [intercellular cell adhesion molecule], VCAM-1 [vascular cell adhesion molecule], (D44) qui se localisent en regard de leurs contrerécepteurs leucocytaires (respectivement LFA-1 [lymphocyte function-associated antigen 1] ou MAC-1 [macrophage-1 antigen, $\mathrm{CD} 1 \mathrm{lb} / \mathrm{CD} 18$, intégrine $\alpha M \beta 2]$, VLA-4 [very late antigen, intégrine $\alpha 4 \beta 1$ ], CD44) [9]. Dans le cas des cellules tumorales métastatiques et des précurseurs neuraux, la seule expression de CD44 est suffisante pour induire la formation d'une coupe de migration bona fide, riche en CD44 comme attendu, mais aussi en ICAM-1 et VCAM-1, même en l'absence de leurs contre-récepteurs (Figure 2C) (S. Cazaubon, observations personnelles et [8]). Ces molécules d'adhérence cellulaire sont toutes associées au cytosquelette d'actine corticale par les protéines de la famille ezrinemoésine-radixine (ERM) ; le recrutement de l'une d'elles au niveau des prolongements endothéliaux formant la coupe de migration pourrait aussi entraîner les autres, via les protéines ERM. II est intéressant de noter que plusieurs agents pathogènes bactériens qui traversent les barrières physiologiques, notamment l'endothélium cérébral, induisent également des réorganisations membranaires qui sont des réminiscences de coupes de migration [10].

L'étape suivant la formation de la coupe de migration est la migration de la cellule à travers l'endothélium ; celle-ci peut être transcellulaire (à travers la cellule endothéliale) ou paracellulaire (entre deux cellules endothéliales) (Figure 2A). La contribution relative de ces deux voies n'est pas claire à ce jour et pourrait dépendre de la localisation du site d'infiltration dans l'arbre vasculaire. Au niveau de l'unité neurovasculaire, une migration leucocytaire par voie transcellulaire a été observée [11] et pourrait constituer un mécanisme majeur, compte tenu de l'obstacle que peut représenter la présence de jonctions serrées entre deux cellules endothéliales cérébrales pour une migration paracellulaire, mais cela reste encore à démontrer $[12,13]$.

Pour conclure, la migration transendothéliale au niveau de l'unité neurovasculaire est un processus finement régulé, non seulement par les cellules endothéliales cérébrales, mais aussi par les cellules périvasculaires qui interviennent dans le maintien de l'intégrité fonctionnelle de la barrière. La migration transendothéliale n'est pas restreinte aux seuls leucocytes, mais peut concerner d'autres types cellulaires présents dans la circulation, en situations pathologiques ou au cours d'une intervention thérapeutique. Finalement, il est intéressant de retenir que la capacité d'une cellule circulante (voire d'un agent pathogène) à induire une coupe de migration semble un prérequis à sa migration à travers la barrière endothéliale. $\bullet$

Transendothelial migration

through the neurovascular unit:

« a cup to cross the barrier »

\section{CONFLIT D'INTÉRÊTS}

Les auteurs déclarent n'avoir aucun conflit d'intérêts concernant les données publiées dans cet article.

\section{RÉFÉRENCES}

1. Daneman R, Zhou L, Kebede AA, Barres BA. Pericytes are required for blood-brain barrier integrity during embryogenesis. Nature $2010 ; 468: 562-6$.

2. Attwell D, Buchan AM, Charpak S, et al. Glial and neuronal control of brain blood flow. Nature 2010; $468: 232-43$.

3. Weiss N, Miller F, Cazaubon S, Couraud PO. The bloodbrain barrier in brain homeostasis and neurological diseases. Biochim Biophys Acta 2008 ; 1788 : 842-57.

4. Neuwelt $\varepsilon A$, Bauer $B$, Fahlke $C$, et al. Engaging neuroscience to advance translational research in brain barrier biology. Nat Rev Neurosci 2011 ; 12 : 169-82.

5. Li B, Zhao WD, Tan ZM, et al. Involvement of Rho/ROCK signalling in small cell lung cancer migration through human brain microvascular endothelial cells. FEBS Lett $2006 ; 580:$ 4252-60.

6. Brazelton TR, Rossi FM, Keshet GI, Blau HM. From marrow to brain: expression of neuronal phenotypes in adult mice. Science $2000 ; 290: 1775-9$.

7. Pluchino $S$, Quattrini A, Brambilla $\varepsilon$, et al. Injection of adult neurospheres induces recovery in a chronic model of multiple sclerosis. Nature 2003 ; 422 : 68894.

8. Rampon C, Weiss N, Deboux C, et al. Molecular mechanism of systemic delivery of neural precursor cells to the brain: assembly of brain endothelial apical cups and control of transmigration by CD44. Stem Cells 2008 ; 26 : 1673-82.

9. Barreiro 0, Yanez-Mo M, Serrador JM, et al. Dynamic interaction of VCAM-1 and ICAM-1 with moesin and ezrin in a novel endothelial docking structure for adherent leukocytes. J Cell Biol 2002 ; 157 : 1233-45.

10. Lemichez $\varepsilon$, Lecuit $M$, Nassif $X$, Bourdoulous $S$. Breaking the wall: targeting of the endothelium by pathogenic bacteria. Nat Rev Microbiol 2010 ; 8 : 93-104.

11. Wolburg H, Wolburg-Buchholz K, Engelhardt B. Diapedesis of mononuclear cells across cerebral venules during experimental autoimmune encephalomyelitis leaves tight junctions intact. Acta Neuropathol 2005 ; 109 : 181-90.

12. Spassky N, Caillé I. La niche neurogénique adulte entre dans la troisième dimension. Med Sci (Paris) $2009 ; 25: 17-8$.

13. Le Cabec V, Van Goethem $\varepsilon$, Guiet R, MaridonneauParini I. La migration des phagocytes : tour d'horizon. Med Sci (Paris) 2011 ; 27 : 1112-20.

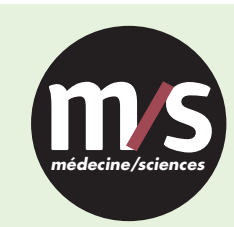

Tarifs d'abonnement $\mathrm{m} / \mathrm{s}-2012$

Abonnez-vous

à médecine/sciences
$>$ Grâce à $m / s$, vivez en direct les progrès des sciences biologiques et médicales

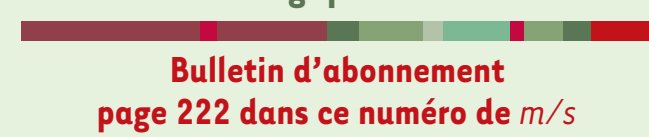

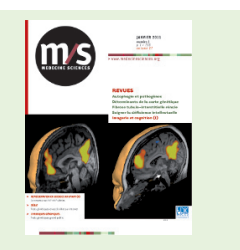

